\title{
Spin Physics Experiments at NICA-SPD with polarized proton and deuteron beams
}

\author{
I.Savin ${ }^{1}$, A.Efremov ${ }^{1}$, D. Peshekhonov ${ }^{1}$, A.Kovalenko ${ }^{1}$, O. Teryaev¹,a, O. Shevchenko ${ }^{1}$, A. Nagajcev ${ }^{1}$, A. Guskov $^{1}$, \\ V. Kukhtin ${ }^{1}$, and N. Toplilin ${ }^{1}$ \\ ${ }^{1}$ JINR, 141980, Dubna, Russia
}

\begin{abstract}
The brief description of the Letter of Intent proposing primarily to perform measurements of asymmetries of the DY pair production in collisions of non-polarized, longitudinally and transversally polarized protons and deuterons which provide an access to all leading twist collinear and TMD PDFs of quarks and anti-quarks in nucleons.
\end{abstract}

Main parts of the Letter of Intent (LoI) [1], devoted to development of spin program of NICA complex at JINR, are related to the studies of the nucleon structure. The beginning of the nucleon structure story refers to the early 50 -ties of the 20th century when in the famous Hofstadter's experiments at SLAC the proton electromagnetic form factor was measured determining thus the proton radius.

The naive quark-parton model (QPM) of nucleons, i.e. of the proton and neutron, has been born. Partons share between themselves fractions, $\mathrm{x}$, of the total nucleon momentum. Parton Distribution Functions (PDFs) are universal characteristics of the internal nucleon structure.

Now the quark-parton structure of nucleons and respectively the quark-parton model of nucleons are becoming more and more complicated and may even lose the universality property. Measurements of the collinear (integrated over $k_{T}$ ) and Transverse Momentum Dependent (TMD) PDFs, the most of which are not well measured or not discovered yet, are proposed in this LoI.

\section{The TMD PDFs.}

The new TMD PDFs can be measured only in the SIDIS or DY processes. In SIDIS, the TMD PDFs can be extracted from the data studying the azimuthal modulations of hadrons which are sensitive to convolution of PDF with the corresponding FF. They include transversity, Sivers, Boer-Mulders, pretzelosity and worm-gear functions.

\section{Physics motivation}

\subsection{The Drell-Yan mechanism as the second microscope of the nucleon structure studies}

The Drell-Yan (DY) process of the di-lepton production in high-energy hadron-hadron collisions is playing an important role in the hadron structure studies.

Applying the Fourier analysis to the measured asymmetries, one can separate each of all ratios of angular modulations coefficients. This will be the ultimate task of the experiments proposed for SPD. The extraction of different TMD PDFs from those ratios is a task of the global theoretical analysis (a challenge for the theoretical community) since each of the angular coefficients is a result of convolutions of different TMD PDFs in the quark transverse momentum space. For this purpose one needs either to assume a factorization of the transverse momentum dependence for each TMD PDFs, having definite mathematic form (usually Gaussian) with some parameters to be fitted, or to transfer functions to impact parameter representation space and to use the Bessel weighted TMD PDFs.

The full set of asymmetries gives the access to all eight leading twist TMD PDFs. However, sometimes one can work with integrated asymmetries. Integrated asymmetries are useful for the express analysis of data and checks of expected relations between asymmetries. They are also useful for model estimations and determination of required statistics.

At NICA the pd- and dd-collisions will be investigated as well. As it is known from COMPASS experiment, the SIDIS asymmetries on polarized deuterons are consisted with zero. But asymmetries in Dp collisions (accessible only at NICA) are expected to be non-zero.

\footnotetext{
${ }^{a}$ Speaker; e-mail: teryaev@
} 


\subsection{New nucleon PDFs and $J / \Psi$ production mechanisms}

The production of lepton pairs in the $q \bar{q}$ processes, via $J / \Psi$ with its subsequent decay into leptons, is analogous to the DY production mechanism. The analogy is correct if the $J / \Psi$ interactions with quarks and leptons are of the vector type. This analogy is known under the name 'duality model'. It may be tested by checking that tensor polarization of $J / \Psi$ is a transverse one. For the TMD PDF studies, the duality model can predict a similar behavior of asymmetries in the lepton pairs production calculated via DY and via $J / \Psi$ events. This similarity follows from the duality model ideas. At NICA such a comparison can be performed at various colliding beam energies.

\subsection{Direct photons}

Direct photon productions in the non-polarized and polarized pp (pd) reactions provide information on the gluon distributions in nucleons. The total cross section of the direct photon production in the pp-collision at $24 \mathrm{GeV}$ via the Compton scattering (according to PYTHIA 6.4) is equal to $1100 \mathrm{nb}$, while the cross section of the qq annihilation is about $200 \mathrm{nb}$. So, the gluon Compton scattering is the main mechanism of the direct photon production and can be used also for extraction of the polarized gluon distribution (Sivers gluon function) from measurement of the transverse single spin asymmetry

\subsection{Spin-dependent reactions in heavy ion collisions}

One of the most interesting quasi-optical effects, the birefringence phenomenon for deuterons (or other particles with spin $J \geq 1$ ) passing through matter, has recently become the area of research. Birefringence occurs when spin particles pass through isotropic non-polarized matter and is due to the inherent anisotropy of particles (as distinct from spin-1/2 particles). The birefringence effect leads to the rotation of the beam polarization vector when a nonpolarized deuteron beam passes through a non-polarized target. Moreover, the appearing spin dichroism effect (the different absorption of deuterons in states with $|\mathrm{m}|=1$ and $\mathrm{m}=0$ ) gives rise to a tensor polarization of the initially non-polarized deuteron beam that has passed through the non-polarized target

The experiments using carbon targets and deuterons with a momentum of $5 \mathrm{GeV} / \mathrm{c}$ were performed at Nuclotron-M accelerator. The birefringence phenomena can be further studied at NICA:

- in few-nucleon systems involving protons and deuterons;

- appearing through the interaction of protons or deuterons with heavy nuclei;

- for heavy nuclei.

- with vector particles produced in inelastic collisions.

The measurement of global polarization of hyperons is also planned and will be compared with CMPQ (Chromomagnetic Polarization of Quarks) model [1] and anoma- lous mechanism [2]. This studies are planned also at other NICA detectors: MPD and BM@N.

\subsection{Future DY experiments on nucleon structure in the world}

The SPD experiments, proposed at the second intersection point of the NICA collider, will have a number of advantages for DY measurements related to nucleon structure studies. These advantages include:

- operations with pp, pd and dd beams,

- scan of effects on beam energies,

- measurement of effects via muon and electronpositron pairs simultaneously,

- operations with non-polarized, transverse and longitudinally polarized beams or their combinations.

Such possibilities permit for the first time to perform comprehensive studies of all leading twist PDFs of nucleons in a single experiment with minimal systematic errors.

\section{Requirements to the NUCLOTRON-NICA complex}

The mentioned research program requires definite characteristics of beams and technical infrastructure. Proton and deuteron beama polarizations both at MPD and SPD: longitudinal and transversal. Absolute values of polarizations during the data taking should be $90-50 \%$. The life time of the beam polarization should be long enough. Measurements of Single Spin and Double Spin asymmetries in DY require running in different beam polarization modes: UU, LU, UL,TU, UT,LL ,LT and TL (spin flipping for every bunch or group of bunches should be considered).

The infrastructure of the Nuclotron-NICA complex should include:

- source(s) of polarized (non-polarized) protons and deuterons,

- system of the beams polarization control and absolute measurements $(3-5 \%)$,

- system of luminosity control and absolute measurements, including a space inside the NICA ring for the beam polarimeters,

- system(s) for transferring data on the beam(s) polarization and luminosity to the experiments.

The infrastructure tasks should be subjects of the separate project(s)

The area of $\pm 3 \mathrm{~m}$ along and across of the beams second intersection point, where the detector for the spin physics experiment will be situated, must be free of any collider elements and equipment. The beam pipe diameter in this region should be less than $10 \mathrm{~cm}$. In this case the angular acceptance of SPD will be close to $4 \pi$. The walls of the beam pipe in the region $\pm 1 m$ of the beams intersection should have a minimal thickness and made of the low- $Z$ material, e.g. beryllium.

\section{Polarized beams at NICA}

The NICA complex at JINR has been approved in 2008 assuming two phases of the construction. The first phase, 
is being realized now, includes construction of facilities for heavy ion physics program while the second phase should include facilities for the program of spin physics studies with accelerated polarized protons and deuterons.

\subsection{Layout of the complex}

The main elements of NICA complex include: the heavy ion source and source of polarized ions, proton and deuteron, with corresponding Linacs, existing superconducting accelerator Nuclotron upgraded to Nuclotron M, new superconducting Booster synchrotron, new collider NICA with two detectors, as well as experimental hall for fixed target experiments with beams extracted from $\mathrm{Nu}$ clotron $\mathrm{M}$.

\subsection{Source of polarized ions and injector}

The new polarized ion source is being commissioned now. It was designed and constructed as a universal pulsing high intensity source of polarized deuterons and protons based on a charge- exchange plasma ionizer. The output D (H) current of the source is expected to be at the level of 10 $\mathrm{mA}$. The expected polarization is about $90 \%$ in the vector for $\mathrm{D}$ and $\mathrm{H}$ and tensor for $\mathrm{D}$ modes. The project is carried out in cooperation with INR of RAS (Moscow). The available from the CIPIOS ion source (IUCF, Bloomington, USA) is partially used. The source will deliver the pulsed polarized proton or deuteron beam with intensity up to $\sim 2 \cdot 10^{11}$ per pulse and repetition rate of $1 \mathrm{~Hz}$.

\subsection{Acceleration of polarized protons and deuterons}

Acceleration of polarized deuterons at the Synchrophasotron was achived for the first time in 1984 and at Nuclotron in 2002 . There are no dangerous spin resonances which could occur during the polarized deuterons acceleration in Nuclotron up to the kinetic energy of $5.6 \mathrm{GeV} / \mathrm{u}$. This limit is practically very close to the maximum design energy of the Nuclotron. There are no doubts about the realization of the project in this case. The only problem in case of deuterons is changing the polarization directions from longitudinal to transversal or backward.

According to the initial NICA project, Nuclotron as the strong focusing synchrotron should accelerate polarized protons from the injection energy $(20 \mathrm{MeV})$ up to the maximum design value of $12.6 \mathrm{GeV}$. For the successful crossing of numerous spin resonances in Nuclotron, the inserted devices like 'siberian snakes' will be designed and installed into the accelerator lattice.

\subsection{NICA in the polarized proton and deuteron modes}

The novel scheme of the polarization control at NICA, suitable for protons and deuterons, is based on the idea of polarized beams manipulation in the vicinity of the zero spin tunes. This approach is actively developed at JLAB for the 8-shaped ring accelerator project. The zero spin tune is a natural regime for the just mentioned case. To provide zero spin tune regime at the collider of the racetrack symmetry, it is necessary to install two identical siberian snakes in the opposite straight sections. In this scheme any direction of the polarization is reproduced at any azimuth point after every turn. However, if one fixes the longitudinal (or vertical) polarization at SPD, the polarization vector at MPD will be rotated by some angle with respect to the direction of the particle velocity vector. This angle depends on the beam energy. If the direction of the polarization is fixed at MPD, some arbitrary polarization angle will occur at SPD. The control insertions can correct this angle.

The number of particles reaches a value about $2.2 \cdot 10^{13}$ in each ring and the peak luminosity $2 \cdot 10^{32} \mathrm{~cm}^{-2} \mathrm{~s}^{-1}$ at 12.7 $\mathrm{GeV}$.

\subsection{Polarimetry at Linac, Nuclotron and NICA}

Requirements to the polarization monitoring and measurements at NICA are the following:

- polarimeters should be installed at several key points of the NICA complex, namely: after Linac, inside the $\mathrm{Nu}$ clotron ring, at the beam transportation line to the collider and in both rings of the collider;

- evaluation of the polarization should be at the standard level for deuterons and protons;

- absolute calibration of the beam polarization should be possible;

- optimal use of the same experimental equipment at different places is desirable;

- permanent monitoring of the beam polarization is necessary.

\section{Requirements to the spin physics detector (SPD)}

Requirements for SPD are motivated by physics and, first of all, by a topology of events and particles to be detected. SPD should operate at the highest possible luminosity. So, all the SPD sub-detectors should have high rate capabilities and preserve high efficiency during a long time.

\subsection{Possible layout of SPD}

Preliminary considerations of the event topologies required SPD to be equipped with the following subdetectors covering $\sim 4 \pi$ angular region around the beam intersection point: vertex detectors, tracking detectors, electromagnetic calorimeters, hadron and muon detectors. Some of them must be in the magnetic field for which there are two options: either toroid or solenoid type.

The most obvious technology for the vertex detector (VD) is a silicon one. It is approved for the MPD VD. Several layers of double sided silicon strips can provide a precise vertex reconstruction and tracking of the particles before they reach the general SPD tracking system. The 
design should use a small number of silicon layers to minimize the material budget.

There are several candidates for a tracking system: multiwire proportional chambers (MWPC), conventional drift chambers and their modification, thin wall drift tubes (straw chambers). The drift chambers are the good candidates for tracking detectors in the end-cap parts of SPD, while straw chambers are the best for the barrel part. Two groups have developed the technology of straw chamber production at JINR with two-coordinate reed-out.

The latest version of the electromagnetic calorimeter (ECAL) module, developed at JINR for the COMPASS-II experiment at CERN, can be a good candidate for ECAL in the barrel and end-cap parts of SPD. The module utilises new photon detector, Avalanche Multichannel Photon Detector (AMPD). AMPD can work in the strong magnetic fields. The modules have rectangular shape but can be produced also in the projective geometry which is better for SPD.

A system of mini-drift chambers interleaved with layers of iron and called the Range System (RS) is developed at JINR for FAIR/PANDA. It can be used in the barrel part of SPD as a hadron and (or) muon detector for the Particle IDentification system (PID).

The hadron and muon detectors in the end-cap parts of SPD are to be identified. As candidates for these detectors the COMPASS muon wall can be considered. It consists of two layers of mini-drift chambers with a block of absorber between them. The more elegant system for hadron and muon detectors of SPD can be constructed using calorimeters suggested for the future linear collider. The prototype of the calorimeter module is under the tests.

\section{Proposed measurements with SPD}

We propose to perform measurements of asymmetries of the DY pairs production in collisions of polarized protons and deuterons which provide an access to all collinear and TMD PDFs of quarks and anti-quarks in nucleons. The measurements of asymmetries in production of $J / \Psi$ and direct photons will be performed simultaneously with DY using dedicated triggers. The set of these measurements will supply complete information for tests of the quarkparton model of nucleons at the twist-two level with minimal systematic errors.

\section{Time lines of experiments}

At the first step of the project it is reasonable to start measurements with non-polarized protons (pp) and with nonpolarized deuterons (dd), (pd). These data would provide a cross checks of our results with very precise world data on PDFs. At the same time new data on the Boer-Mulders PDF will be obtained.

At the second step the measurements should be performed with longitudinally polarized protons and deuterons in pp, pd and dd collisions with the beam polarizations UL, LU, LL to obtain asymmetries $A_{L U}, A_{U L}$ and $A_{L L}$ in each case. These data will be cross checked by existing data on $g_{1}$ and provide new information on the Worm-gear-L PDF in proton and neutron.

At the third step (the most important) measurements should be performed with transverse beam polarization in pp, pd and dd collisions ( $U T, T U$ and $T T$ ) to obtain asymmetries $A_{U T}, A_{T U}$ and $A_{T T}$ in each case. These data will be cross checked by existing data on Transversity PDF and provide new information on the Sivers, Worm-gear-T and Pretzelosity PDFs in proton and neutron.

Finally, at the fourth step (the most difficult) measurements should be performed with pp, pd and dd beams when one beam is polarized longitudinally while the other one is polarized transversally in order to measure asymmetries $A_{L T}$ and $A_{T L}$ in each case. These data will provide new information and cross checks of our results on Transversity, Worm-gear-L, Pretzelosity and Worm-gearT PDFs.

\section{Conclusions}

The current LoI is the core of the studies of hadrons spin structure at NICA. The SPD program in the course of preparing the proposal will be linked to that of MPD and $\mathrm{BM} @ \mathrm{~N}$, providing the coherent spin physics program at NICA.

\section{References}

[1] I. A. Savin, A. V. Efremov, D. V. Peshekhonov, A. D. Kovalenko, O. V. Teryaev, O. Y. Shevchenko, A. P. Nagajcev and A. V. Guskov et al., arXiv:1408.3959 [hep-ex].

[2] M. Baznat, K. Gudima, A. Sorin and O. Teryaev, Phys. Rev. C 88 (2013) 061901 [arXiv:1301.7003 [nucl-th]]. 